\title{
Transparent and Efficient Shared-State Management for Optimistic Simulations on Multi-core Machines
}

\author{
Alessandro Pellegrini \\ Roberto Vitali \\ Sebastiano Peluso \\ Francesco Quaglia \\ pellegrini@dis.uniroma1.itrvitali@dis.uniroma1.it peluso@dis.uniroma1.it quaglia@dis.uniroma1.it \\ High Performance and Dependable Computing Systems Research Group \\ DIIAG, Sapienza, University of Rome
}

\begin{abstract}
Traditionally, Logical Processes (LPs) forming a simulation model store their execution information into disjoint simulations states, forcing events exchange to communicate data between each other. In this work we propose the design and implementation of an extension to the traditional Time Warp (optimistic) synchronization protocol for parallel/distributed simulation, targeted at shared-memory/multicore machines, allowing LPs to share parts of their simulation states by using global variables. In order to preserve optimism's intrinsic properties, global variables are transparently mapped to multi-version ones, so to avoid any form of safety predicate verification upon updates. Execution's consistency is ensured via the introduction of a new rollback scheme which is triggered upon the detection of an incorrect global variable's read. At the same time, efficiency in the execution is guaranteed by the exploitation of non-blocking algorithms in order to manage the multi-version variables' lists. Furthermore, our proposal is integrated with the simulation model's code through software instrumentation, in order to allow the application-level programmer to avoid using any specific API to mark or to inform the simulation kernel of updates to global variables. Thus we support full transparency. An assessment of our proposal, comparing it with a traditional message-passing implementation of variables' multi-version is provided as well.
\end{abstract}

\section{INTRODUCTION}

A traditional way to achieve high performance simulations is the employment of Parallel Discrete Event Simulation (PDES) techniques [1]. They are based on the partitioning of the simulation model into Logical Processes (LPs) that can execute events in parallel on different CPUs and/or different CPU-Cores, and rely on synchronization mechanisms to achieve causally consistent execution of simulation events.

As it is well recognized, the optimistic synchronization approach, namely the Time Warp protocol [2], which is based on rollback for recovering possible timestamp-order violations due to the absence of block-until-safe policies for event processing, is likely to favor speedup in general application/architectural contexts. In particular, it has been shown to exhibit performance relatively independent of the lookahead of the specific simulation model, and has also been shown not to suffer (in terms of amount of rollback in the parallel execution) from non-minimal message delivery latency.

On the other hand, supporting the Time Warp protocol, while still guaranteeing a simple and flexible application programming model, is not trivial. In particular, being Time Warp based on concepts related to state recoverability, the level of transparency towards the application programmers depends on the extent and the mode according to which state recoverability functionalities operate within the Time Warp platform. Recent achievements along this direction (see, e.g., [3]) have enabled fully transparent and performance-optimized recoverability via state logs for LPs making use of dynamic memory for the representation of their states, and possibly relying on thirdparty libraries (thus software external to the application layer) for performing state updates during event processing. At the same time, alternative attempts to the semi-automated generation of reverse code for backward-computation-based state reconstruction have been also presented (see, e.g., [4]). Such kind of approaches extremely simplify the job of the application programmers since no state-management task in relation to synchronization (e.g., state-log tasks) requires to be implemented at the application level.

Actually, most of the solutions tackling transparency have been oriented to the original definition of the Time Warp protocol [2], where the LPs' states are assumed to be disjoint. Hence, according to this definition, each LP is only allowed to modify its private state variables upon processing new events, and the interactions (namely inter-dependencies) across LPs are only allowed to be instantiated via cross-LP scheduling of simulation events. On the other hand, having different LPs sharing (at least a portion of) the state of the simulation model may result in a more flexible paradigm, whose relevance has been fully recognized as a crucial issue in the development of parallel simulation applications [5], [6].

In this article we tackle the issue of transparently and efficiently supporting shared-state in optimistic simulation systems run on top of shared-memory/multi-core machines, by enabling the application programmer to access within the event processing routine both the private state of the LP and a global portion of the state, whose instance is represented by the value of global variables admitted within the application-level code. Overall, with our solution the programmer is allowed to rely on the heap for allocating/deallocating memory chunks belonging to the private state of each LP, as already supported via the approach in, e.g., [3], while also being able to rely on global variables for the shared portion of the state thanks to the innovative solution we provide in this paper.

We implemented a fully-featured shared-state management system targeted at IA-32/x86-64 architectures and ELF executables. Also, we have integrated such system within the open-source ROOT-Sim (ROme OpTimistic Simulator) package [7] which implements an optimistic run-time environment supporting ANSI-C compliant application-level software implementing the LPs' logic in the form of event-handlers.

In order to provide efficient support for the management of shared-state variables, in terms of both forward and backward computation, our proposal relies on an application-transparent multi-version scheme based on non-blocking access/update 
operations. This allows improving the level of parallelism when the shared-state is accessed by multiple LPs concurrently scheduled on different CPU-cores.

The results of an experimental assessment of the sharedstate management architecture are also reported for the case of a wireless system simulation application run on top of ROOTSim on an HP ProLiant server equipped with 32 CPU-cores and 32 GB of RAM memory.

The remainder of this paper is structured as follows. In Section II we discuss related work. The shared-state management architecture is presented in Section III. Section IV presents experimental data aimed at assessing the pragmatical viability of our proposal.

\section{RELATED WORK}

The work in [8] discusses how state sharing might be emulated by using a separate LP hosting the shared data and acting as a centralized server. To tackle performance issues, the work proposes to modify the rollback behavior of this special LP by introducing the notion of version records. This is an approach similar to the one proposed in [6], where a theoretical presentation of algorithms to implement a Distributed Shared Memory mechanism is presented in terms of protocols to keep replicated instances of a variable coherent. In particular, one of the provided algorithms proposes to realize variables as multi-version lists where write operations install new version nodes and read operations find the most suitable version. Although this approach shows similarities to ours, read and write operations are mapped to message passing primitives, which is instead not the case for our proposal. This places a hard burden on the centralized node(s), which in the case of a simulation model performing frequent read/write operations on shared variables can produce a non-sustainable overhead. Additionally, these approaches are strongly oriented to distributed simulation environments, while we target the trend of shared-memory/multi-core machines.

A further enhancement has been presented in [9], where the notion of state query is introduced. An LP needing a portion of the state which belongs to a different LP can issue a query message to it, and wait for a reply containing the suitable value. In case this value is later detected to be no longer valid, an anti-message $\left({ }^{1}\right)$ is sent so to invalidate the query. Again, this approach relies on message passing, and is not transparent to the application programmer.

The work in [10] proposes to integrate supports for sharedstate in terms of global variables, by basing the architecture on [11]. Although this proposal supports in-place read/write operations as we do (i.e., LPs directly access the only copy of the data, avoiding a commit phase at the end of the execution of an event), they provide no transparency, as the applicationlevel code must explicitly register an LP as a reader/writer on the shared variables, and furthermore the synchronization between LPs accessing shared variables is based on locks, while we provide a non-blocking implementation.

\footnotetext{
${ }^{1}$ In Time Warp, an anti-message is a negative copy used to annihilate a previously sent message, namely an already scheduled event. Anti-messages are used to propagate the effects of causality errors across the LPs by retracting events scheduled during the causal inconsistent portion of the simulation.
}

In the context of the High-Level-Architecture (HLA), proposals for supporting shared-state can be found in [12], [13]. These proposals are again targeted at a distributed environment, since they are based on a middleware component which relies on a timestamp-ordering approach for implementing a request/reply protocol. Additionally, these approaches are targeted at the conservative synchronization protocol, where there is no need to detect and handle causality violations, while we target optimistic synchronization.

The Software Transactional Memory (STM) paradigm [14] allows multiple threads to access global information while ensuring consistency wrt concurrent accesses. The main differences between multi-version-based STMs [15] and our proposal lie in that (i) STM does not enforce transparency wrt the application-level programmer, since transactions must be explicitly marked; (ii) write operations do not work inplace, i.e., data updating is performed on separate buffers (i.e., write-sets) which are then copied (i.e., externalized) into the global buffer after some safety predicate is computed during the commit phase; (iii) when an update is externalized, it cannot be undone, i.e., there is no need for supporting rollback operations on externalized values, as instead it may occur in the optimistic synchronization protocol for parallel simulators.

The work in [16] proposes a framework targeted at multicore machines and based on Time Warp, where Extended Logical Processes (Ex-LP), defined as a collection of LPs, can access state variables of each other directly. Every ExLP should therefore manage an event-list to perform rollback operations due to shared data accesses. In addition, public attributes are referred to variables which can be accessed by LPs in other Ex-LPs. The work proposes to handle shared attributes accesses by relying on a specifically-targeted STM implementation, where events are mapped to transactions and the actual implementation of the STM is based on [10]. This proposal inherits most of the features of the general STM paradigm, so that our proposal is set aside this one.

As for non-blocking algorithms, avoiding mutual exclusion has been considered a benefit since the early 1970's [17]. Lamport [18] gave the first non-blocking algorithm for the problem of a single-writer/multiple-reader shared variable. Herlihy [19] proved that for non-blocking implementations of most interesting data types (linked lists among them), a synchronization primitive that is universal, in conjunction with reads and writes, is both necessary and sufficient. A universal primitive is one that can solve the consensus problem [20] for any number of processes. In our implementation we rely on Compare\&Swap (CAS), which is a universal primitive. The work in [21] presents the implementation of a non-blocking linked list, which we have readapted for our own purposes.

A subtle problem associated with most lock-free algorithms is the ABA problem. It was first reported in association with the introduction of the CAS instruction on the IBM System 370 [22]. It occurs when a thread $\mathrm{T} 1$ reads a value $\mathrm{A}$ from a shared object and then an interrupting thread T2 modifies the value of the shared object from A to B and then back to A. When T1 resumes, it erroneously assumes that the object has not been modified. Given such behavior, there is a serious risk that T2's execution is going to violate the correctness of the object's semantic. Practical solutions to the ABA problem 
include the use of hazard pointers [23] or the association of a version counter to each element in platforms supporting a double-word compare-and-swap primitive (CAS2) such as IA32 [24]. We explicitly rely on the latter solution to avoid the ABA problem in our non-blocking implementation.

\section{Shared-State MAnAgement ARChitecture}

Being our approach targeted at multi-core machines, in our Shared-State Management Subsystem (SSMS) we have explicitly decided to rely on shared memory for keeping the current state of global variables. This allows a fast access to the data structures, although requiring some sort of synchronization between instances in order to ensure correctness. To leverage the synchronization burden, we have decided to implement data structures' accesses as non-blocking algorithms [25], which are expected to ensure better performance than locking ones when accesses are statistically spread across the various portions of the data. To ease the application-level programmer, we have addressed transparency via software instrumentation, so that no additional API or code construct should be used to notify SSMS of accesses to global variables.

In this section, we provide a detailed description of the architectural choices and the motivations behind each key component of SSMS. Additionally, we will discuss the reduced set of APIs provided by SSMS, which allow a fast integration into any optimistic simulation platform adhering to the optimistic synchronization protocol.

\section{A. Read/Write Detection}

In order to provide complete transparency to the applicationlevel programmer, accesses in read/write mode to global variables must be explicitly intercepted. To this end, we rely on instrumentation techniques aimed at modifying the actual instructions executed by software executables, without altering their actual semantics. In particular, in the work in [26] we presented a versatile Instrumentation Tool (IT) targeted at IA-32/x86-64 instruction sets [24], [27] and ELF executables [28], on GNU/Linux Operating Systems. By relying on IT, at compile time the application-level instruction code (i.e., the assembly bytestream) is modified in order to replace operations loading data to and from memory with actual function calls which are the entry points of our SSMS. These entry points are associated with the following APIs provided by SSMS: write_global_variable(void *orig_addr, time_type lvt, ...) and void *read_global_variable (void *orig_addr, time_type my_lvt). They allow accessing the versions within the version lists for a given variable at a certain Logical-Virtual-Time (LVT).

We have identified two main groups of instructions/code blocks which have to be handled within the applicationlevel assembly code. First, in IA-32 simple load and store operations are identified by mov instructions. Whenever IT's parser identifies a mov instruction, it is analyzed in order to determine whether it is targeting memory as a source or destination operand, and a call to write_global_variable or read_global_variable is replaced accordingly. When the mov instruction involves a load operation from memory, an additional postamble to the function call is placed, in order to have the actual value returned by read_global_variable placed into the correct CPU register where the application-level software is expecting the value to be found.

Second, the IA-32 instruction set provides more complex instructions which allow an executable to efficiently modify memory areas in-place. As a relevant example, we propose instructions like ADD m32, r32 or INC m32. In this case, IT replaces the instructions with a block of instructions, entailing a couple of calls to the SSMS's read and write APIs, and re-implementing the same logic with several CPU instructions. This implementation of course adds some overhead, nevertheless it allows to integrate our SSMS completely transparently wrt the application-level programmer.

High-level programming languages allow to access memory objects in a non-direct way, namely through the use of pointers. Since IT works at compile time, it is not possible to statically determine whether a pointer will target a global variable or not. To cope with this issue, we use IT to instrument any mov instruction which can handle pointers through a call to a monitor function which fastly determines if a pointer targets a global variable. In particular, at compile time, via the usage of a custom ld-based linker script we insert symbols called_bss_start,_bss_end, _data_start, _data_end, within the application-level ELF executable, which mark off the area containing global variables. Upon a call to the monitor routine, a fast check on these boundaries is performed. If a pointer falls within this area, the operation is redirected to SSMS, on the other hand the original mov instruction is executed.

As a last note, Intel's instruction set provides string instructions which allow to perform operations on memory buffers instead of single memory locations. In particular, movs and stos instructions allow the program to copy or modify large buffers at once. In order to cope with the presence of these complex instructions, SSMS provides two additional APIs, namely copy_buffer () and set_buffer () which simulate the execution of these operations on version lists if they are found to target global variables (e.g., global arrays). Otherwise, they just execute the original movs or stos operations. Therefore, at compile time, IT replaces every string operation involving memory update with a function call to these APIs, accordingly.

The last operation we perform at compile time is the inspection of the application-level ELF object file in order to extract information concerning global variables. In particular, by exploring the application object we extract from the symbol table . symtab all the STT_OBJECT / STT_COMMON symbols and store their name, address and size in a text file which will be later used at startup time for setting up the version lists. In this way, by exploiting the 〈name, address, size〉 tuple, we are able to transparently identify any access to global variables which will be likely used by the applicationlevel code during the execution of the simulation model, allowing the programmer to rely on the complete set of constructs provided by ANSI-C. We note that, although there will be more instances of the simulation kernel running the application-level code, a global variables' address is a common information shared among the instances, as long as its virtual 
address will be the same and is cabled into the executable.

\section{B. Accounting for Third-Party Libraries}

The possibility to rely on third-party libraries depends on whether they will be invoked on global variables or not. We have explicitly addressed the case of read/write operations performed by third-party software, just focusing on stdlib. Specifically, SSMS provides a set of function wrappers for all those functions which produce in-memory accesses by the application-level software through pointers passing. The wrappers simply check whether global variables are involved in the operation. In this case, operations are redirected to SSMS APIs for accessing version lists. Otherwise, the original stdlib functions are called.

\section{Memory Map and Version Lists}

As hinted before, SSMS explicitly targets sharedmemory/multi-core machines. In order to significantly enhance performance, we have decided to avoid requesting to the underlying operating system shared memory segments ondemand, whenever SSMS needs to install some data structure. On the other hand, at simulation startup the master kernel installs a large shared memory segment, and broadcasts to other kernel instances its id. The shared segment is partitioned according to the definition of the following structure:

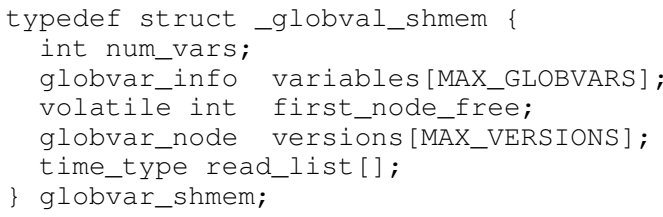

In particular, the shared memory segment is divided into several fixed-sized portions. One portion, namely variables, is an array which is used to manage global variables. Upon initialization of SSMS, the configuration text file described in Section III-A is loaded and parsed. The field num_vars is used to keep track of how many variables are actually handled, and for each of them an entry in the variables array is populated. To allow a fast retrieval of the global variables, we use a fast hash function to determine which entry in the variables array will store the information associated with a specific variable. In particular, the position in the array is determined with a fast bitwise operation - namely, address \& $(\sim(-$ MAX_GLOBVARS $))$ - since MAX_GLOBVARS is set to be a power of two. In case collisions are found, separate chaining is used as a means for finding a free place. Each entry in the variables array is structured as:

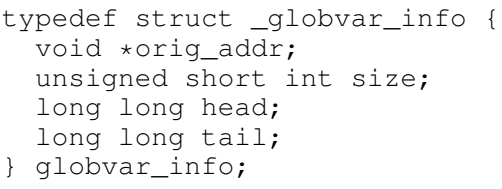

orig_address stores the global variable's original address, which is used as hash table's key; size describes which is the size (in bytes) of the global variable.

Since we are preallocating shared memory, version lists must be implemented using nodes scattered around the preallocated segment. In particular, versions is an array of

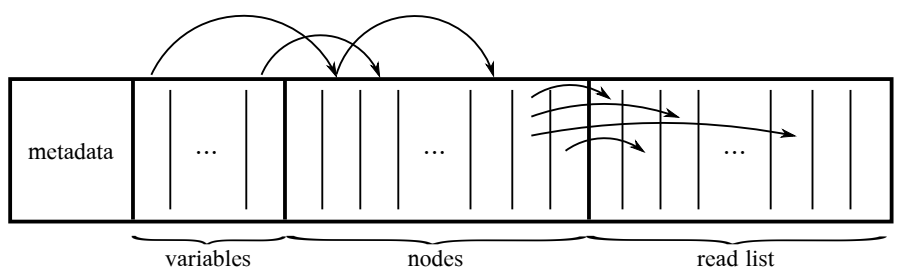

Fig. 1: Preallocated Shared Memory Map

fixed-sized nodes which can be used for any list, and head and tail are indices within this array, which is composed of entries structured as follows:

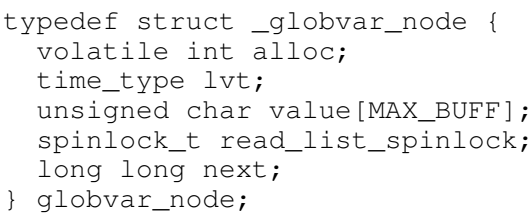

where lvt is the logical time associated with the version, value is the global variable's value, and next is used to identify which is the following node in the list. A node can therefore be seen as a snapshot of the state of a single global variable at a certain LVT. In Figure 1 we provide a complete picture of the preallocated memory map.

Node versions' entries can belong to any list, and given that lists are accessed without the use of locks, a special allocation function must be used, ensuring that no two simulation kernel instances running concurrently are given the same entry for handling two different versions.

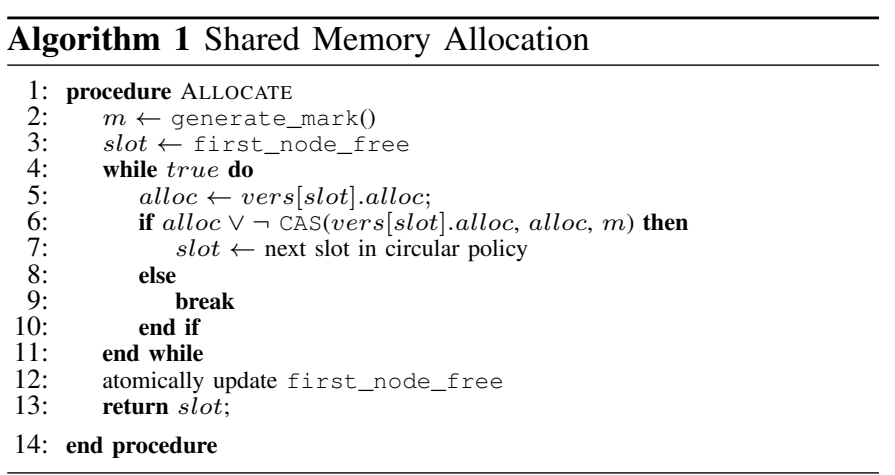

The Allocate pseudocode is given in Algorithm 1. In order to allow concurrent accesses, it relies on CAS $\left({ }^{2}\right)$, which allows to update involved data only if no other process has updated the same data in the meanwhile. The globvar_shmem data structure holds in first_node_free the value of the first element of the versions array to start trying to allocate from. Its manipulation is based on the classical algorithm used by the LINUX kernel for managing the bitmap of file descriptors associated with a process. Specifically, it is always atomically increased upon allocation, and gets atomically decreased in case an entry is released having index less than the first chunk currently available within that block. Starting

\footnotetext{
${ }^{2}$ In particular, we rely on the IA-32's cmpxchg. Throughout this paper we mention atomic operations which are implemented directly in assembly using native atomic instructions.
} 


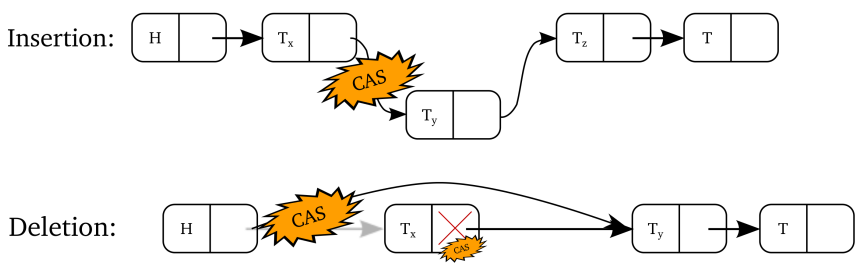

Fig. 2: Non-Blocking Linked List Operations

from that slot, a kernel instance tries to allocate a node by storing via a CAS operation a non-zero value within the alloc field of globvar_node, which tells whether a node is currently in use. In case the CAS fails, the next node in the array is selected and the procedure is repeated, until it eventually succeeds $\left({ }^{3}\right)$. The companion function RELEASE is much simpler, as it only entails resetting the alloc and updating first_node_free, via an atomic_set call.

In order to cope with the ABA problem [22], we have explicitly decided to consider a node allocated if the alloc field is non-zero. In particular, we store into it a unique value every time a node is allocated, so that two allocations can be identified as different. The macro generate_mark produces an integer value which is in turn composed of two short integers, one holding the unique id of a kernel instance and the other holding the value of a per-kernel counter which is incremented every time the macro is invoked $\left({ }^{4}\right)$.

Once a node is allocated, it gets organized into a nonblocking linked list, which is implemented according to a modified version of the one proposed in [21]. Concurrent insertions are handled via the use of a single CAS operation, which is used to introduce the newly allocated node into the list by acting on the next field of the predecessor node. As for deletion, two CAS are used, one to mark the next field of the deleted node as logically deleted, and another to physically delete the node. We have slightly modified the algorithm in order to take into account our specific needs. In particular, the FIND-NODE procedure has been augmented in order to return the alloc field, to explicitly cope with the ABA problem, and the INSERT procedure does not fail if a node with the same key (i.e. LVT) already exists. Specifically, the new node is simply linked after the originally existing one. In addition, we note that LPs are more likely to access versions associated with higher LVTs, since well partitioned/balanced optimistic simulations usually proceed relatively evenly. Therefore, we sort the versions in the lists in descending order, to avoid a complete scan of the list every time we want to find a node in it.

To avoid the ABA problem in linked lists, pointers (i.e. indices) to nodes are composed (every time they are updated) by a unique mark generated via the aforementioned macro generate_mark and the real index, allowing to capture

\footnotetext{
${ }^{3}$ To check if the space is up, a counter of available free nodes is kept as well in shared memory, which is managed via an atomic_decrement operation.

${ }^{4}$ generate_mark can of course return two equal values when the counter overflows, but this situation can happen after a significant simulation time, so we consider it to be statistically non-significant for the ABA problem.
}

the situation where two nodes are still adjacent but one was deallocated and then reallocated during the execution of the non-blocking algorithm by different kernel instances.

The operations performed on the versions lists are depicted in Figure 2.

\section{Accessing Version Lists}

The APIs offered by SSMS provide two main functions to access global variables, namely read_global_variable and write_global_variable, which we will refer to as READ and WRITE from now on.

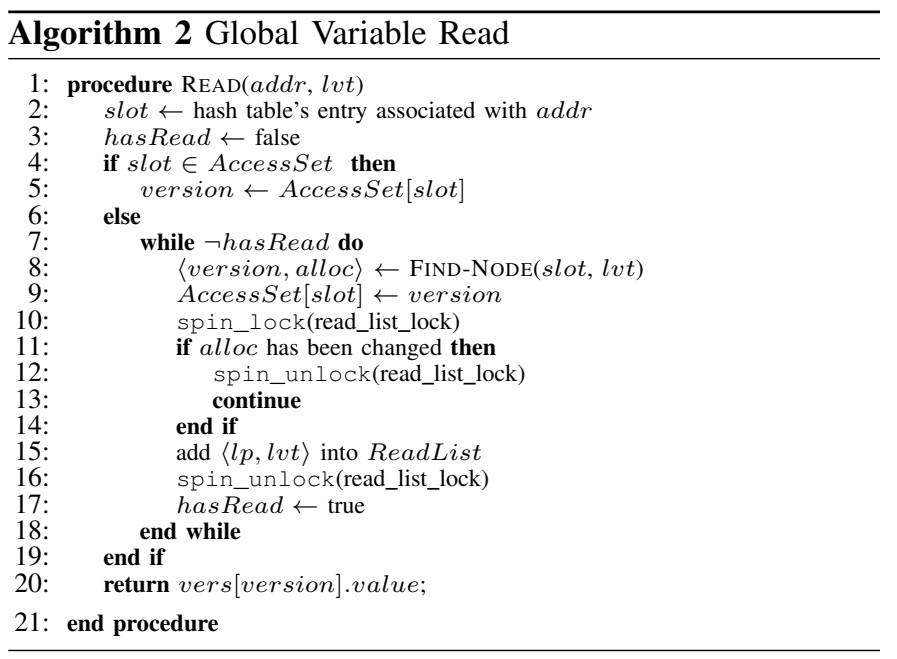

READ operation's pseudocode is provided in Algorithm 2. For efficiency reasons, before letting an LP execute a simulation event, SSMS sets up an AccessSet, i.e., a mapping between version nodes and variables. Whenever a variable is accessed for the first time, FIND-NODE ${ }^{5}$ ) determines which is the most suitable version for the given LVT, and a couple $\langle$ slot, version $\rangle$ is placed into AccessSet in order to speedup the retrieval of the version, avoiding the scan of the list upon subsequent accesses.

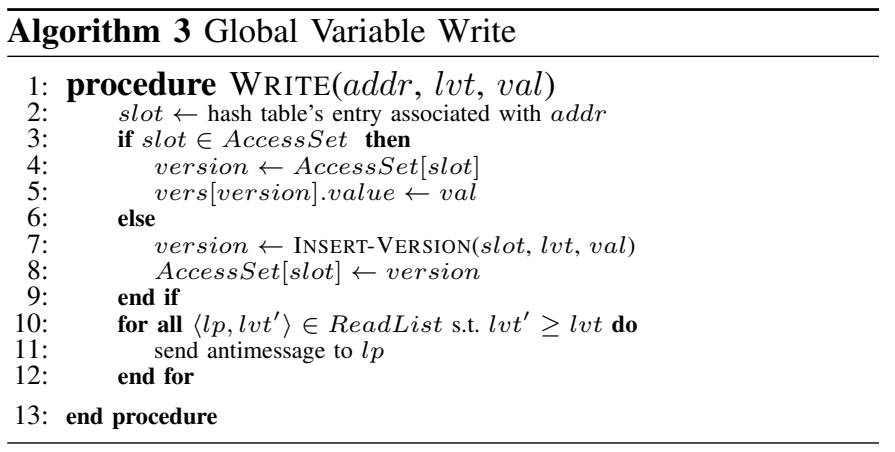

As for the WRITE operation, the pseudocode of which is presented in Algorithm 3, its behavior is twofold depending on whether it is invoked for the first time since the beginning

${ }^{5}$ We remind that FIND-NODE is a modified version of the one presented in [21]. For a detailed description of the procedure, we throw back to that work. In addition, we note that a version node is always available, even before any WRITE operation, since at startup the initial value of the global variable is placed into the version list. 


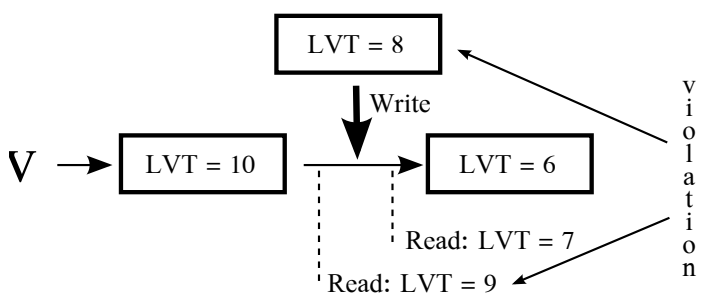

Fig. 3: Occurrence of the Rollback Operation

of the current event's execution. In particular, upon the first access on a variable, the Access Set for that particular event is populated. Otherwise, a call to INSERT-VERSION is performed which, as stated in Section III-C, creates a new version. The second part of the WRITE operation entails checking the ReadList for ensuring consistency, as it will be clearly depicted in Section III-E.

\section{E. Synchronization and Rollback Operations}

In order to strengthen the optimism of our implementation, we allow interleaved reads and writes on a version list, and we explicitly avoid a version $k$ installed at LVT $t_{k}$ to invalidate every version $j$ such that $t_{k}<t_{j}$. In fact, we note that consistency is violated only if, at LVT $t_{x}$ an LP reads the version associated with LVT $t_{y}$ such that $t_{y} \leq t_{x}$, and at a certain point during the execution a new version node associated with LVT $t_{z}$ such that $t_{y} \leq t_{z}<t_{x}$ is installed.

This means that every process which reads a certain version node must leave a mark of that operation, i.e., visible reads [29] are enforced. In fact, as shown in Figure 3, we are interested in undoing only the events which read a version older than the new one which has just been inserted.

To this end, we augment the classical notion of rollback as presented by the Time Warp synchronization protocol, by sending a special anti-message to all the LPs which have read a so-defined causally inconsistent version after any write operation. This is reflected into Algorithms 2 and 3. In fact, in the READ operation, before returning the variable's value, the tuple $\langle l p, l v t\rangle$ is inserted into the ReadList for that particular version. This operation is included within a specially designed critical section to ensure consistency. In fact, a spinlock for that particular ReadList is taken, ensuring that no other process will start the rollback operation while the ReadList is being updated. Otherwise, this scenario would produce a non-trackable read operation. In addition, after the spinlock has been taken, a check on the variation of the alloc field for that particular version is performed, so to avoid the ABA problem due to a critical race between the deallocation/allocation procedure and the ReadList update. At the same time, at the end of the WRITE operation, the ReadList of the left node is checked in order to find all the LPs which read the previous node's value, while they were requesting a version at an LVT such that they should have read the one in the version which was just installed. Although the list is linked in only one direction, given the implementation of FIND-NODE, locating the previous node is immediate.

We note that another step must be undertaken in order to ensure correctness. In particular, whenever a special antimes- sage is received because of an inconsistent read, any version node installed due to that particular event must be removed. To this end, we augmented the concept of message quеие and modified the WRITE function so that whenever a node is installed during the execution of an event, the message queue keeps track of this operation via a pointer to the node created during the event's execution. In case a rollback operation entails the undoing of that event, the node is removed from the version list, and the ReadList is scanned for sending antimessages to every LP which read that particular node.

\section{F. Memory Recovery}

In Time Warp, the notion of fossil collection is defined, i.e., the process of recovering memory by deleting simulation state snapshots which are no longer needed. In particular, at a periodic rate, the Global Virtual Time (GVT) is computed as the minimum timestamp of not yet processed events or intransit messages/antimessages in the whole simulation system. Since during the execution of an event an LP can schedule a new event at an LVT which is equal to, or greater than, the one associated with the event being executed, there cannot be a rollback operation involving a simulation state snapshot associated with a timestamp less than the GVT. Therefore, any snapshot belonging to a logical time window before the GVT can be discarded.

In our proposal, we extend the notion of fossil collection by defining the version list pruning. In particular, upon GVT computation, the version lists associated with global variables are scanned in order to find which is the first node $i$ stamped with $t_{i} \leq G V T$ and that node is selected as the barrier node. Any node marked with a timestamp $t_{k}<t_{i}$ is marked as free and removed from the list. For implementations where there is no actual event processing during GVT computation, the version list pruning is thread safe, and can therefore be executed efficiently, with no need to synchronize the access. In particular, the various lists can be divided evenly across the various kernel instances, and each kernel performs the memory recover executing in isolation. This choice provides a more efficient execution and still ensures correctness.

\section{EXPERIMENTAL DATA}

\section{A. Test-Bed Application}

The hardware architecture used for testing our proposal is a 64-bit NUMA machine, namely an HP ProLiant server, equipped with four $2 \mathrm{GHz}$ AMD Opteron 6128 processors and 64GB of RAM. Each processor has 8 cores (for a total of 32 cores) that share a $12 \mathrm{MB}$ L3 cache (6 MB per each 4cores set), and each core has a $512 \mathrm{~KB}$ private L2 cache. The operating system is 64-bit Debian 6, with Linux Kernel version 2.6.32.5. The compiling and linking tools used are gcc 4.3.4 and binutils (as and $1 \mathrm{~d}$ ) 2.20.0.

We have run our model on top of the ROme OpTimistic Simulator (ROOT-Sim) [7], which is an open-source, generalpurpose simulation platform developed using C/POSIX technology, based on a simulation kernel layer that ultimately relies on MPI for data exchange across different kernel instances, and which adheres to the optimistic synchronization paradigm. Interaction with the application-level software is handled via a simple and reduced API, while LPs' state management and 
recoverability is offered by DyMeLoR [26], [30], a memory manager which allows rollbackable dynamic memory allocation and release by the application, performed via hooked standard malloc library calls, offering full transparency.

As a test-bed, we have used Personal Communications Service (PCS), a suite of differently parameterized simulation models of wireless communication systems adhering to GSM technology. The different parameterization entails variations of the transmission capabilities offered by each cell, as well as variations of the call arrival rate. In the employed simulation models, wireless communication channels are modeled in a high fidelity fashion via explicit simulation of power regulation/usage and interference/fading phenomena (implemented according to the results in [31]) on the basis of the current state of the corresponding cell (also expressed as a function of current meteorological conditions).

Upon the start of a call destined to a mobile device currently hosted by a given wireless cell, a call-setup record is instantiated via dynamically-allocated data structures, which gets linked to a list of already active records within that same cell. Each record gets released when the corresponding call ends or is handed-off towards an adjacent cell. In the latter case, a similar call-setup procedure is executed at the destination cell. Upon call-setup, power regulation is performed, which involves scanning the aforementioned list of records for computing the minimum transmission power allowing the current call-setup to achieve the threshold-level SIR value. Data structures keeping track of fading coefficients are also updated while scanning the list, according to a meteorological model defining climatic conditions (and related variations). The employed simulation models have been developed for execution on top of ROOT-Sim in a way that each LP models a single wireless cell. Hence, the event-handler callback involves the update of individual cells' states, and cross-LP events are essentially related to hand-offs between different cells.

Calls inter-arrival time is exponentially distributed, and average duration is set to 2 minutes. The expected rate for call inter-arrival has been set to achieve channel utilization factor on the order of $15 \%$, while the residence time of an active device within a cell has a mean value of $5 \mathrm{~min}$ and follows the exponential distribution.

To evaluate the efficiency of our proposal, we have extended the simulation model having a set of global variables handling global statistics. In particular, upon each events execution the total number of calls, the total number of handoffs, and the global cumulated power is updated in the shared state. In addition, we have re-implemented the model in order to have a centralized LP keeping in its disjoint simulation state the global attributes. Every LP willing to update a shared attribute issues a message request to the centralized LP, which in turn sends back the current value. Any update on the current value is then sent as another message to the centralized LP.

For the above scenario, we have run experiments with 64 wireless cells, modeled as hexagons covering a square region, each one managing 1000 wireless channels. We have measured the cumulated event rate (expressed as the amount of cumulated committed events per Wall-Clock-Time unit), which is a classical indicator of the speed of the optimistic simulation run.

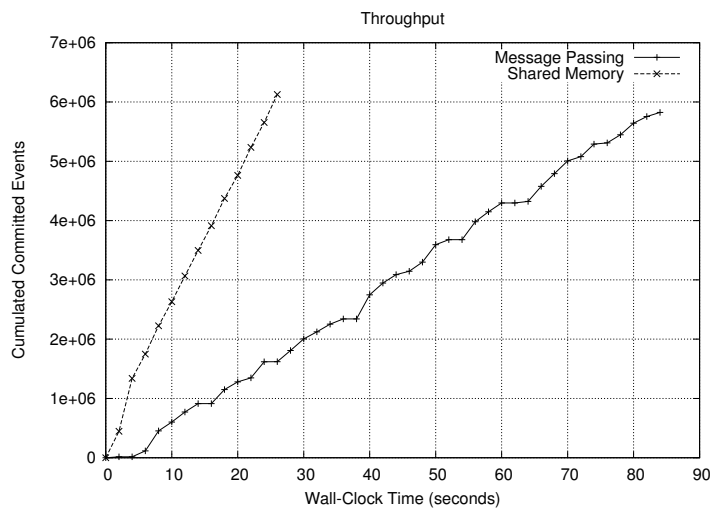

Fig. 4: Throughput Running on 32 Kernel Instances

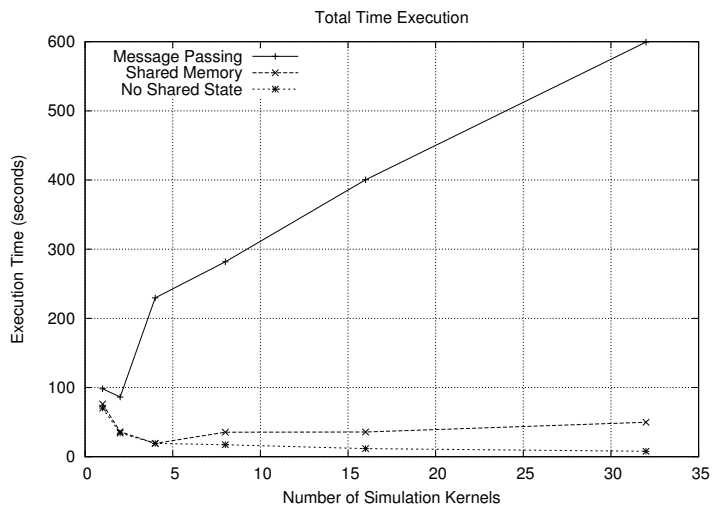

Fig. 5: Scaling wrt the Number of Parallel Instances

\section{B. Results}

In Figure 4 we present the throughput associated with our proposed test-bed model run on top of 32 simulation kernel instances, each one running on a private CPU-core of our test machine. By the results, we can see that the execution of the simulation model relying on our SSMS provides a speedup in the order of $70 \%$. In addition, we note that there is a tangible difference between the two curves' trends. In fact, the throughput associated with the SSMS execution has a constant growth, which suggests a constant event commitment rate. On the other hand, the centralized-LP implementation's slope shows fluctuations, which are related to the large amount of events associated with variables' reads/updates which must be processed. Therefore, the number of committed events per GVT interval is not constant, due to the fact that the amount of workload processed by differentiated LPs is totally different and that the LVT of the LP keeping the shared state diverges from the other LPs' one (this can entail a higher rollback probability), a scenario which is not present at all when relying on the multiversion lists in the shared memory version case.

At the same time, Figure 5 shows the total execution time of the simulation wrt the number of parallel simulation kernel instances on which the model is run. In addition to the set of experiments described before, we present also the curve associated with another implementation of the benchmark, where the shared attributes are kept in the disjoint LPs' simulation 
states and are reduced at the end of the simulation. By the results, we can see that both the SSMS and the centralizedLP implementation suffer from some form of thrashing. In fact, the centralized-LP version provides a speed-down in the order of $100 \%$ when the model is parallelized on top of 4 parallel kernel instances, while SSMS shows the same behaviour starting from 8 parallel kernel instances. The version with no shared state shows a trend which is the one expected by a parallel simulator.

We note that in this configuration, the SSMS's speedup wrt the centralized-LP is very large. Of course, the overhead in the centralized-LP case could be leveraged by having different LPs handle different variables, but this solution would not scale well wrt the size of the shared state in the simulation model.

Finally, we note that the simulation model used to assess the validity of our proposal is a worst case for our architecture, since at every event's execution some updates on the global variables are performed, producing a large contention on the linked lists. A simulation model which relies on shared-state for synchronization rather than for global statistics would benefit much more from the proposed architecture.

\section{Conclusions}

In this work we have presented the design/implementation of an efficient support to shared-state for optimistic simulation platforms, targeted at multi-core/shared-memory architectures. We have explicitly relaxed the classic simulation models' state coinstraints, allowing the usage of portion of shared states among simulation objects, relying on global variables. We have provided the application-level programmer with full transparency, and we have exploited intrinsic carachteristics of our target architecture to enhance synchronization performance by relying on a non-blocking implementation.

\section{REFERENCES}

[1] R. M. Fujimoto, "Parallel discrete event simulation," Communications of the ACM, vol. 33, no. 10, pp. 30-53, Oct. 1990.

[2] D. R. Jefferson, "Virtual Time," ACM Transactions on Programming Languages and System, vol. 7, no. 3, pp. 404-425, 1985.

[3] R. Vitali, A. Pellegrini, and F. Quaglia, "Autonomic log/restore for advanced optimistic simulation systems," International Symposium on Modeling, Analysis, and Simulation of Computer Systems, vol. 0, pp. 319-327, 2010.

[4] C. D. Carothers, K. S. Perumalla, and R. Fujimoto, "Efficient optimistic parallel simulations using reverse computation," ACM Transactions on Modeling and Computer Simulation, vol. 9, no. 3, pp. 224-253, Jul. 1999.

[5] R. M. Fujimoto, "Featured article - parallel discrete event simulation: Will the field survive?" INFORMS Journal on Computing, vol. 5, no. 3, pp. 213-230, 1993

[6] H. Mehl and S. Hammes, "How to integrate shared variables in distributed simulation," SIGSIM Simulation Digest, vol. 25, no. 2, pp. 14 41, Sep. 1995

[7] F. Quaglia, A. Pellegrini, and R. Vitali, "ROOT-Sim: The ROme OpTimistic Simulator: http://www.dis.uniroma1.it/ hpdes/root-sim/," Oct. 2011.

[8] D. Bruce, "The treatment of state in optimistic systems," SIGSIM Simulation Digest, vol. 25, no. 1, pp. 40-49, Jul. 1995.

[9] A. Fabbri and L. Donatiello, "SQTW: a mechanism for state-dependent parallel simulation. description and experimental study," in Proceedings of the 11th Workshop on Parallel and Distributed Simulation, jun 1997, pp. $82-89$.
[10] K. Ghosh and R. M. Fujimoto, "Parallel discrete event simulation using space-time memory," in Proceedings of the 1991 International Conference on Parallel Processing, 1991, pp. 201-208.

[11] K. M. Chandy and R. Sherman, "Space-time and simulation." University of Southern California, Information Sciences Institute, 1989, pp. 53-57.

[12] B. P. Gan, M. Low, J. Wei, X. Wang, S. Turner, and W. Cai, "Synchronization and management of shared state in hla-based distributed simulation," in Proceedings of the 2003 Winter Simulation Conference, vol. 1, dec. 2003, pp. $847-854$ Vol.1.

[13] M. Y. H. Low, B. P. Gan, J. Wei, X. Wang, S. J. Turner, and W. Cai, "Shared state synchronization for hla-based distributed simulation," Simulation, vol. 82, no. 8, pp. 511-521, Aug. 2006.

[14] N. Shavit and D. Touitou, "Software transactional memory," in Proceedings of the 14th annual ACM symposium on Principles of distributed computing. New York, NY, USA: ACM, 1995, pp. 204-213.

[15] J. Cachopo and A. Rito-Silva, "Versioned boxes as the basis for memory transactions," Sci. Comput. Program., vol. 63, no. 2, pp. 172-185, Dec. 2006.

[16] L. li Chen, Y. shuai Lu, Y. ping Yao, S. liang Peng, and L. da Wu, "A well-balanced time warp system on multi-core environments," in Proceedings of the 2011 IEEE Workshop on Principles of Advanced and Distributed Simulation, june 2011, pp. 1 -9.

[17] W. B. Easton, "Process synchronization without long-term interlock," SIGOPS Operating Systems Review, vol. 6, no. 1/2, pp. 95-100, Jun. 1972.

[18] L. Lamport, "Concurrent reading and writing," Commun. ACM, vol. 20, no. 11 , pp. 806-811, 1977.

[19] M. Herlihy, "Wait-free synchronization," ACM Transactions on Programming Languages and Systems, vol. 13, pp. 124-149, January 1991.

[20] M. J. Fischer, N. A. Lynch, and M. S. Paterson, "Impossibility of distributed consensus with one faulty process," $J$. ACM, vol. 32, no. 2, pp. 374-382, Apr. 1985.

[21] T. Harris, "A pragmatic implementation of non-blocking linked-lists," in Distributed Computing, ser. Lecture Notes in Computer Science, J. Welch, Ed. Springer Berlin / Heidelberg, 2001, vol. 2180, pp. 300314.

[22] I. B. M. Corporation, IBM System/370 Extended Architecture, Principles of Operation. IBM Publication No. SA22-7085, 1983.

[23] M. M. Michael, "Hazard pointers: Safe memory reclamation for lockfree objects," IEEE Trans. Parallel Distrib. Syst., vol. 15, no. 6, pp. 491-504, Jun. 2004.

[24] IA-32 Intel(R) Architecture Software Developer's Manual, Volume 2A: Instruction Set Reference, A-M, Intel Corporation.

[25] M. Herlihy and N. Shavit, "On the nature of progress," in Proceedings of the 15th International Conference on Principles of Distributed System, 2011, pp. 313-328.

[26] A. Pellegrini, R. Vitali, and F. Quaglia, "Di-DyMeLoR: Logging only dirty chunks for efficient management of dynamic memory based optimistic simulation objects," in Proceedings of the 23rd Workshop on Principles of Advanced and Distributed Simulation. IEEE Computer Society, 2009, pp. 45-53.

[27] IA-32 Intel(R) Architecture Software Developer's Manual, Volume 2B: Instruction Set Reference, $N$-Z, Intel Corporation.

[28] The Santa Cruz Operation and AT\&T, "System V Application Binary Interface," Tech. Rep., 1997.

[29] J. Burns and N. A. Lynch, "Mutual exclusion using invisible reads and writes," in In Proceedings of the 18th Annual Allerton Conference on Communication, Control, and Computing, 1980, pp. 833-842.

[30] R. Toccaceli and F. Quaglia, "DyMeLoR: Dynamic memory logger and restorer library for optimistic simulation objects with generic memory layout," in Proceedings of the 22nd Workshop on Principles of Advanced and Distributed Simulation. IEEE Computer Society, 2008, pp. 163172.

[31] S. Kandukuri and S. Boyd, "Optimal power control in interferencelimited fading wireless channels with outage-probability specifications," IEEE Transactions on Wireless Communications, vol. 1, no. 1, pp. 46$55,2002$. 